\title{
CalVal Evaluation of DESIS products in Amiaz Plain and Makhtesh Ramon Test sites, Southern Israel.
}

\author{
Daniela Heller Pearlshtien * and Eyal Ben-Dor
}

Remote Sensing Laboratory, Geography Department, Porter School of Environment and Earth Science, Faculty of Exact Science, Tel Aviv University, Tel-Aviv 699780, Israel; bendor@tauex.tau.ac.il

*Correspondence: dh@mail.tau.ac.il

ISPRS Commission and Working group- Commission I WG I/1 Multi- and Hyperspectral Sensing

KEYWORDS: Hyperspectral, Calibration, Validation, Test site.

\begin{abstract}
:
The new era of hyperspectral remote (HSR) sensors in orbit is approaching. Missions such as CHIME of the European Space Agency (ESA), EMIT/SBG of NASA, EnMAP of the German Aerospace Center (DLR), and SHALOM of the Israel Space Agency (ISA) will launch in the near future, while other HSR sensors are already in orbit, such as DESIS of DLR, PRISMA of the Italian Space Agency (ASI), and HISUI of the Japan Aerospace Exploration Agency (JAXA). Vicarious calibration (VC) of satellite sensors is vital to tracking a sensor's performance during its lifetime and is a routine procedure in any satellite mission. Accordingly, searching for ideal sites for CAL/VAL operation is an important task that should be part of the mission planning. This study demonstrates two areas in southern Israel that can be acquired in one overpass as VC sites: Amiaz Plain (AP) and Makhtesh Ramon (MR), which were evaluated for their fulfillment of all VC requirements for HSR sensors. AP ( $5 \mathrm{~km}^{2}$ of homogeneous bright target) was found suitable for radiometric calibration, and MR $\left(200 \mathrm{~km}^{2}\right)$ for spectral and thematic validations. We checked the applicability of these sites using a high-end airborne HRS sensor (AisaFENIX 1K sensor with 420 bands, 375-2500 nm spectral range, and 1.5-m spatial resolution) along with comprehensive field studies and ground measurements. Accordingly, we developed an operational VC protocol to use these sites for both radiometric and spectral quality inspection of HRS satellites. We demonstrated this capability on recent PRISMA and DESIS reflectance products. Here we provide these analyses and recommend how to use these areas to further examine DESIS data's performance. We call for collaborations with individuals and space agencies in using these VC sites, where we will provide ground-truth information and fulfill any other requirements for VC.
\end{abstract}

\section{INTRODUCTION}

Accurate radiometric and spectral values of any sensor in orbit are crucial factors that affect the ability to extract quantitative data from the image (Teillet, 2015). Data products are susceptible to spectral band variations after a sensor launch. If the spectral bands have changed position or width, or there are uncertainties about their characteristics, this has a direct impact on the radiometric quality, affecting the output products (e.g., Suits et al., 1988; Teillet et al., 1990; Flittner and Slater, 1991; Obata et al., 2017; Kabir et al., 2020). The radiometric and spectral calibration of the sensor degrades with time due to launch stresses, electronic and material aging, and effects related to conditioning of the near-Earth orbit (Müller, 2014). Hyperspectral remote sensing (HRS) radiometric and spectral information is even more susceptible than multispectral sensors, as the technology is based on high spectral resolution and precise physical data (Thenkabail, 2016). There are several ways to calibrate the sensor during the lifetime of a mission. The vast majority of EO missions use on-board calibration means, and the most common is vicarious calibration (VC), which uses ground references from test sites. This type of calibration is applied over a well-known ground site that is stable in space and time, and is measured during (or close in time to) the sensor overpass, to estimate at-sensor radiance or top of the atmosphere (TOA) reflectance. Comparisons of these estimations with image-based values provide post-launch monitoring of the radiometric calibration (e.g., Robert and Kaufman, 1986; Biggar et al., 1994; Scott et al., 1996; Secker et al., 2001; Teillet, 2015; Yoshida et al., 2005; Bouvet et al., 2019). This approach was introduced in the early 1980s and has since been improved to keep pace with the evolution of the radiometric requirements of the sensors (Bouvet et al., 2019).
Criteria for terrestrial VC site selection for passive remote sensing have been well-documented (Scott et al., 1996): spatial uniformity over a large area (within 3\%), flat spectral reflectance across solar-reflective wavelengths, temporally invariant surface properties (within $2 \%$ ), arid region with a low probability of cloud and vegetation cover. It is also essential that the characterization of any given calibration reference site be well-represented by the measurement site's spectral properties (radiance, reflectance, weather conditions). Based on the high importance of VC and the forthcoming era of high-end HSR sensors in orbit, several groups are focusing on VC activity (for example, the IEEE P4001 group, GEO group (group on earth observation), and the Calibration and Validation Working Group (CVWG) of NASA's for HSR SBG satellite.) Several sites have already been documented and archived by the Committee on Earth Observation Satellites (CEOS) (Cal/Val Home - CalValPortal, n.d.). http://calvalportal.ceos.org/home.

Due to the importance of getting reliable physical data from remote sensing sensors, More terrestrial sites are being investigated for use as reference standard test sites for postlaunch sensor calibration. Thus, specialists in the international community are endeavoring to assemble databases of worldwide calibration facilities. The most famous is RadCalNet, which consists of four radiometric calibration-instrumented sites located in the USA, France, China, and Namibia, and is the result of efforts by the RadCal NetWorking Group under the umbrella of the CEOS Working Group on Calibration and Validation (WGCV) and Infrared Visible Optical Sensors (IVOS) (Bouvet et al., 2019).

Today, there are a few HSR programs in orbit, with others planned. The DESIS hyperspectral instrument is one of them, was built by Teledyne Brown Engineering (TBE; Alabama, 
USA) and serves as the primary instrument operator while the German Aerospace Center (DLR) is in charge of the instrument calibration and the processors. The DESIS sensor operates from the International Space Station (ISS). It is a push-broom imaging spectrometer that is spectrally sensitive over the visible and near-infrared (VNIR) range from 400 to $1000 \mathrm{~nm}$; it has 235 bands with a spectral sampling distance of $2.55 \mathrm{~nm}$ and 30 m GSD (ground sample distance) (Krutz et al., 2019). It is also important to mention that another HSR sensor that covers the $400-2500 \mathrm{~nm}$ spectral range is mounted on the ISS, namely the HISUI from the Japan Aerospace Exploration Agency JAXA (HISUI, n.d.) (Kamei et al., 2011). And, in 2022, NASA EMIT's will join them and be also mounted on the ISS. Other operational HSR sensors are: PRISMA of the Italian Space Agency (ASI) (238 spectral bands) (PRISMA, n.d.) (Candela et al., 2016), India's Hyperspectral Imaging Satellite HySIS (256 bands) ("HySIS," n.d.), and China's Advanced Hyperspectral Imager AHSI (330 bands) (Liu et al., 2020). In addition, a few more hyperspectral missions are planned: the EnMAP from DLR (Environmental Mapping and Analysis Program of 30-m GSD, 420-2450 nm, 242 bands) (Guanter et al., 2015), CHIME of the European Space Agency (ESA) (30-m GSD, 400-2500 nm), and SHALOM of ASI and the Israel Space Agency (ISA) (Feingersh and Dor, 2015) (9-m GDS, 220 bands, 400-2500 nm). With this new era of hyperspectral sensors, post-launch calibration, both radiometric and spectral in high demand and high priority for all space agencies.

Within the arid climatic region of Israel, several interesting areas in terms of a stable and mostly cloud-free landscape can be used as sites for both radiometric and spectral VC. Among these are Amiaz Plain (AP) and Makhtesh Ramon (MR), located in the Negev Desert in Southern Israel.

The first to recognize the potential of $\mathrm{AP}$ for $\mathrm{VC}$ were Gilead and Karnieli (2004), who examined several areas in Israel for VC of multispectral orbital sensors. AP is part of the Judean Desert Nature Reserve. It is in a very arid area (mean annual rainfall is $47 \mathrm{~mm}$ ) with scarce vegetation, elevation ranging from 260 to $270 \mathrm{~m}$ below mean sea level, with mountain ridges on the western and eastern edges. AP is a homogeneously bright plain consisting of silty carbonate. This area is perfect for radiometric VC performance and calibration. MR is a national geological park and reserve occupying about $200 \mathrm{~km}^{2}$. It is an anticline formation with an eroded central valley, drained mainly by a single creek. Steep walls bound the valley, with friable sandstone at the bottom and more resistant limestone and dolomites at the top. The exposed area is relatively flat and consists of pure mineralogical exposures from different geological ages (from Triassic to Holocene). Since the 1990s, MR has been studied with remote-sensing sensors and found to hold significant spectral features of iron-oxide, clay, gypsum, and carbonate minerals (e.g., Kaufman, 1991; Ben-Dor and Kruse, 1995; Anker et al., 2009; Notesco et al., 2015, 2016; Schmidt and Karnieli, 2001; Heller-Pearlshtien et al., 2021;). MR is thus a perfect site for spectral calibration and a vital area for thematic examination based on stable mineral formations and landscape.

We checked the feasibility of the AP and MR sites with an extensive airborne hyperspectral campaign using the Specim high-end AisaFENIX 1K sensor with 420 bands, 375-2500 nm spectral range, and $1.5-\mathrm{m}$ spatial resolution (Hyperspectral Sensor AisaFENIX - Specim, Spectral Imaging Ltd., n.d.) along with comprehensive field studies and measurements. Accordingly, we developed an operational VC protocol to use these sites for both radiometric and spectral quality inspection of HRS satellites. We created an online MR database website ("Makhtesh Ramon Cal/ Val Site," n.d.) that is being regularly updated and summarizes the campaign and ongoing groundtruth data and results. This information is available for researchers who want to use these areas as the past, present, and future CAL/VAL sites. The capability of these suggested areas to serve as CAL/VAL sites has been recently demonstrated on PRISMA L1 and L2D products (Heller-Pearlstein et al., 2021). This paper provides the performance of DESIS products based on the protocol that we developed and examined for PRISMA using the spectral information acquired from these sites.

\section{MATERIALS AND METHODS}

\subsection{DESIS data cube}

Three images of the MR area from Feb 24 (two images) and Feb 8 (one image) 2021, and one image over AP from Dec 3, 2020, were acquired from DESIS (EOweb GeoPortal, n.d.). We used the L1C radiometric-georectified image and the L2A atmospheric-corrected data cube. Sensor information: 235 bands, spectral range 400-1000 nm, 30-m GSD, full-width half maximum (FWHM) $\sim 3.5 \mathrm{~nm}$ (without binning), $\sim 7.0 \mathrm{~nm}$ (binning 4), swath $30 \mathrm{~km}$, sensor altitude $400 \mathrm{~km}$.

\subsection{PRISMA data cube}

One image of PRISMA over MR from April 2021 was acquired from PRISMA's website (PRISMA, n.d.). The L2D atmospheric correction product was used. Sensor information: 238 bands, range of $400-2500 \mathrm{~nm}, 30-\mathrm{m}$ GSD, FWHM $\leq 12 \mathrm{~nm}$, swath 30 $\mathrm{km}$, sensor altitude $615 \mathrm{~km}$.

\subsection{AisaFENIX data cube}

An airborne campaign using the AisaFENIX 1K airborne sensor (Hyperspectral Sensor AisaFENIX - Specim, Spectral Imaging Ltd., n.d.) over MR and AP (Figure 1) was carried out on Apr 5, 2017, covering the entire MR $200 \mathrm{~km}^{2}$ area (25 flight lines) and AP $5 \mathrm{~km}^{2}$ (one flight line). Sensor information: 420 bands, spectral range 375-2500 nm, 1.5-m GSD, FWHM: visible (VIS) $3.4(\mathrm{~nm})$, NIR-shortwave infrared (SWIR), $6.2(\mathrm{~nm})$, swath $1.8 \mathrm{~km}$, along with extensive ground-truth measurements, including field and geology surveys to calibrate the sensor and validate its mapping products. All of the data are summarized and available in the online MR database ("Makhtesh Ramon Cal/ Val Site," https://storymaps.arcgis.com/stories/bb5bf09ec7414454a012bfe 9bf4b8545 


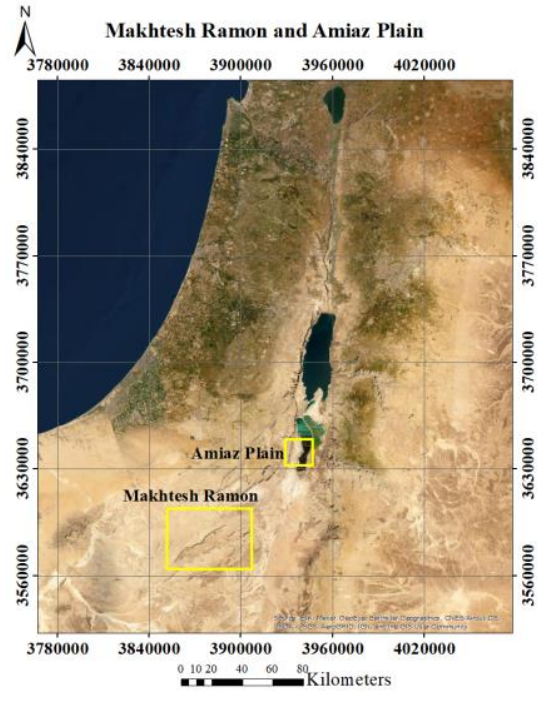

Figure 1. Locations of Makhtesh Ramon and Amiaz Plain in Israel

\subsection{MR test sites}

Six test sites in MR (Figure 2) were chosen to evaluate the spectral calibration of the HSR sensors used in this study. These test sites are stable in space and time and homogeneous flat areas with unique spectral features across the sensors' spectral range. They are very easy to access and close to one another. The test sites encompassed different spectral ranges, as follows: VNIR spectral range 400-1000 $\mathrm{nm}$ (sites 1 and 2): 1. brown questa rich in iron oxides $\left(30^{\circ} 37^{\prime} 14.45\right.$ "N, 34 ${ }^{\circ} 50^{\prime} 29.12$ "E, size $410 \mathrm{~m} \times 140 \mathrm{~m}$ ); 2. laccolite mineral on gypsum soil (30³6'12.23 "N, 3453'42.80 "E, size $410 \mathrm{~m} \mathrm{x} 70 \mathrm{~m})$. SWIR1 $1450-1800 \mathrm{~nm}$ (sites 3 and 4): 3 . gypsum old mine (30 $35^{\prime} 42.24$ "N, 345'ㄴ' 1.13 "E, size $340 \mathrm{~m}$ x $240 \mathrm{~m}$ ); 4. gypsum soil fans $\left(30^{\circ} 36^{\prime} 7.45\right.$ "N, 345'ㄱ' 37.08 "E, size: $500 \mathrm{~m}$ x $\left.200 \mathrm{~m}\right)$. SWIR2 2000-2500 nm (sites 5 and 6): 5. kaolinite old mine (30॰37'19.85 "N, 3451'0.77 "E, size 540 m x $340 \mathrm{~m})$; 6. calcite layer $\left(30^{\circ} 36^{\prime} 19.72\right.$ "N, 3451'49.18 "E, size 320 m x $\left.200 \mathrm{~m}\right)$. Although only two sites capture unique spectral features in the VNIR range, we used all six test sites to validate the DESIS spectral calibration and L2A products.
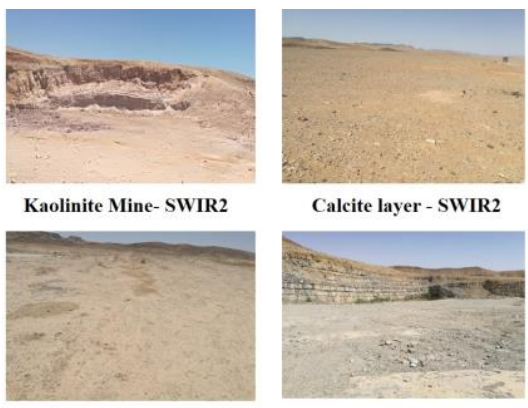

Gypsum Mine- SWIR1

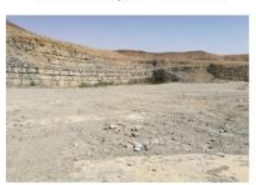

Gypsum Soil-SWIR1

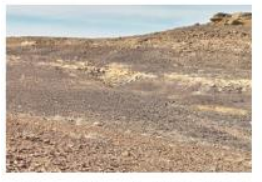

Brown Questa (BQ) -VNIR

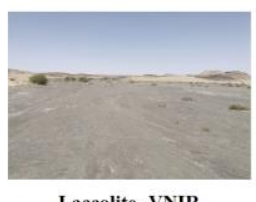

Laccolite -VNIR

Figure 2. Test sites in Makhtesh Ramon

\subsubsection{Evaluation process- MR test sites}

To evaluate the spectral similarity between DESIS and AisaFENIX spectra at the test sites, we resized the AisaFENIX data cube to 30-m GSD and resampled it to the DESIS spectral configuration (235 bands across the VNIR region). The reflectance ratio (Rrn; Eq. 1) and the spectral angle mapper (SAM; Eq. 2) indices (Kruse et al., 1993) were calculated. Lower SAM values indicate higher spectral similarity along with ratio values that are close to 1 . where Rtn is the examined reflectance spectrum, and Rtrn is the reference spectrum of the same target. Rrn represents the ratio between the examined and reference spectra, and $\mathrm{n}$ is the number of wavelengths used.

$$
\begin{aligned}
& R r_{n}=\frac{R t_{n}}{R t r_{n}}, \\
& S A M=\cos ^{-1}\left(\frac{\sum_{i=1}^{n} R t_{n_{i}} R t r_{n_{i}}}{\sqrt{\sum_{i=1}^{n} R t_{n_{i}}^{2}} \sqrt{\sum_{i=1}^{n} R t r_{n_{i}}^{2}}}\right)
\end{aligned}
$$

\subsection{ASD FieldSpec ${ }^{\circledR}$}

The ASD (Analytical Spectral Devices) FieldSpec ${ }^{\circledR}$ was used in the field measurements (model FSP 350-2500 nm). The spectroradiometer has a spectral range of $350-2500 \mathrm{~nm}$ with 2151 bands, with 3-nm and 8-nm resolution for the VNIR and SWIR regions, respectively). More than 30 points were taken along $30 \mathrm{~m}^{2}$ at each test site. To simulate a pure 1 pixel of a 30 meter spatial resolution sensor, we followed the same protocol in each field target site; measuring the reflectance on the center of the field target area in (X) shape, ten measurements for each 30-meter line, then randomly adding 12 more measurements around this $\mathrm{X}$ in four directions. The average of all 32 points represents the test site spectral signal. The GPS coordination is measured in the $\mathrm{X}$ center. All measurements were conducted with a Pistol Grip with a $25^{\circ}$ field of view. A standard white panel (Spectralon ${ }^{\circledR}$ ) was used to calculate the reflectance values.

\section{RESULTS AND DISCUSSION}

This section focuses on the quality performance of the DESIS sensor by examining its radiometric and spectral products compared to those of the well-calibrated AisaFENIX sensor campaign over the MR area. A comparison is also made to another orbital hyperspectral sensor, The Italian ASI's PRISMA.

\subsection{DESIS L1C radiance product}

The TOA radiance model was generated with the MODTRAN $®$ radiance transfer code ("MODTRAN®," n.d.) for AP, using the ASD reflectance of the area.

Atmospheric and solar radiation were generated to yield simulated TOA radiance values for the DESIS overpass (Dec 3, 2020 , azimuth angle $188.9^{\circ}$, zenith angle $53.7^{\circ}$, desert aerosol, sensor height $400 \mathrm{~km})$. The simulated radiance results $(\mathrm{mW}$ 
$\mathrm{cm}^{-2} \mathrm{sr}^{-1} \mathrm{~nm}^{-1}$ ) were compared with the authentic DESIS L1C product signal (Figure 3 ).
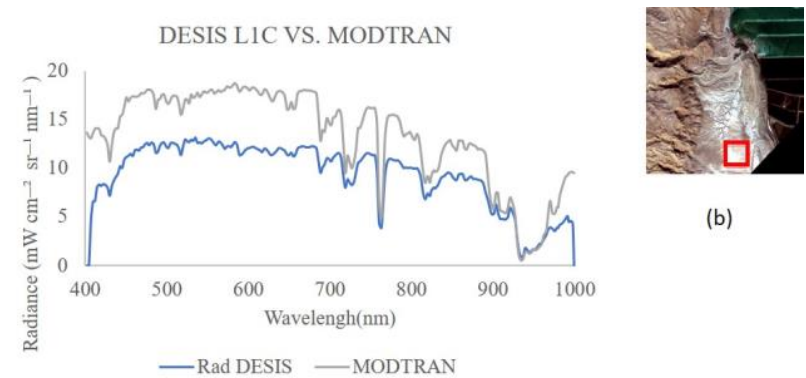

(b)

(a)

Figure 3. a) Comparison of DESIS TOA radiance to MODTRAN simulation. b) Amiaz Plain marked on DESIS image

As seen in Figure 3, both the simulated (MODTRAN) and actual (L1C) radiative responses were similar, with an offset of about $5 \mathrm{~mW} \mathrm{~cm}^{-1} \mathrm{sr}^{-1} \mathrm{~nm}^{-1}$ and with noticeable absorbances at the same bands of atmospheric gases such as oxygen $\left(\mathrm{O}_{2}\right)$ at 688 and $763 \mathrm{~nm}$ and water vapor at $822 \mathrm{~nm}$ and $934-944 \mathrm{~nm}$. It is important to mention that Amiaz's plain elevation is $260 \mathrm{~m}$ below mean sea level. Therefore we added $260 \mathrm{~m}$ to the sensor height $(400+0.260=400.26 \mathrm{~km})$ in the MODTRAN model calculation. This compensation may have influenced the albedo differences we see between the model and DESIS.

\subsection{Comparison of DESIS L2A to AisaFENIX and ASD spectra}

To evaluate the DESIS L2A atmospheric-corrected product, we compared the average spectra of DESIS (approximately 10 pixels marked) at each test site to the ASD spectrometer field measurements and AisaFENIX images after spatial and spectral resampling as described in section 2.4 (Figure 4a,b). Figure 5 provides the ratio between these sensors.

Please note: that the precisions and the high quality of AisaFENIX signal at MR were established and shown in our previous article (Heller-Pearlstein et al., 2021)

Figures 4 and 5 show high similarity between the DESIS spectra, field ASD, and AisaFENIX spectra. However, there is some drift in the DESIS spectra at bands lower than $450 \mathrm{~nm}$. In addition, atmospheric absorbance residuals are still seen in the DESIS reflectance, i.e., the oxygen absorbance at $763 \mathrm{~nm}$ and the water vapor absorbance at $943 \mathrm{~nm}$. This similarity was further calculated over the entire spectral region using the SAM index, and the correlation between DESIS and AisaFENIX along with the calculated RMSE are shown in Table 1.
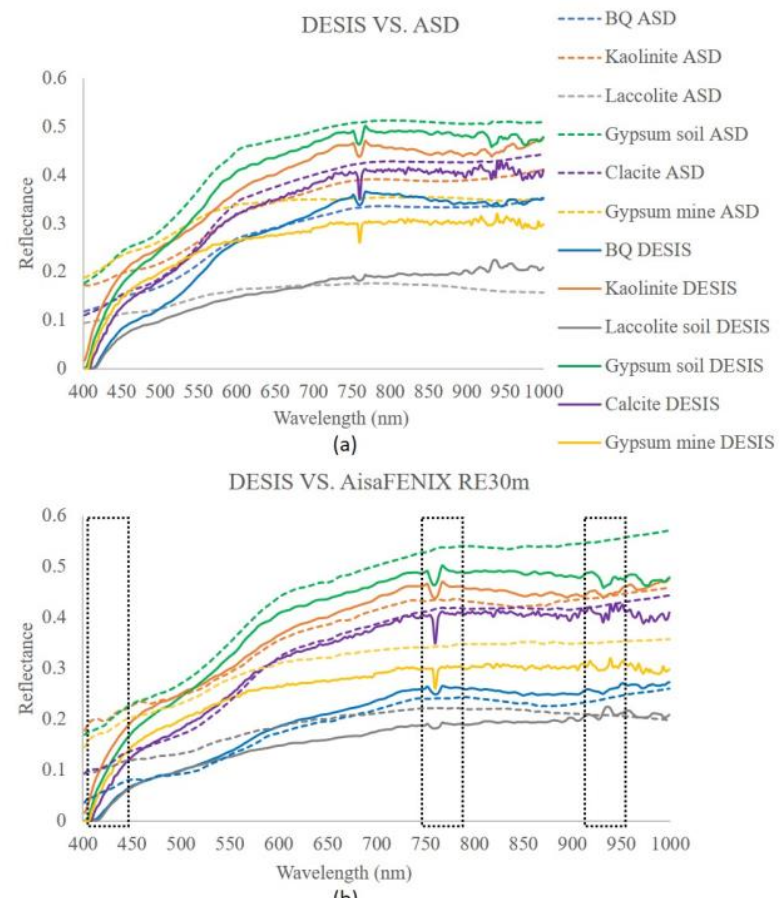

(b)

Figure 4. Comparison of DESIS L2A to ASD (a) and AisaFENIX (b) resampled spectra on the test sites. Solid lines, DESIS reflectance; dashed lines, ASD and AisaFENIX reflectance, accordingly

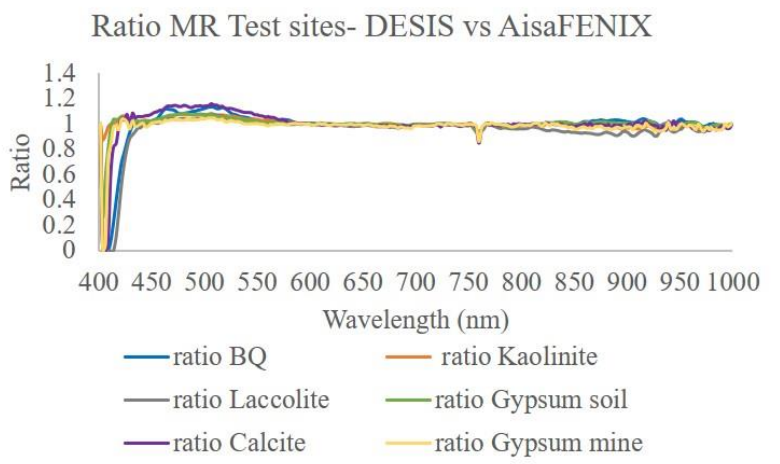

Figure 5. Reflectance) The ratio between DESIS L2A and AisaFENIX

Table 1. Reflectance comparison

\begin{tabular}{|c|c|c|c|}
\hline Test site & SAM & RMSE & $\mathbf{R}^{\mathbf{2}}$ \\
\hline Brown questa & 0.0566 & 0.0179 & 0.9874 \\
\hline Laccolite & 0.1505 & 0.0384 & 0.9036 \\
\hline Gypsum soil & 0.0672 & 0.0566 & 0.9559 \\
\hline Gypsum mine & 0.0593 & 0.0526 & 0.9351 \\
\hline Kaolinite & 0.0437 & 0.0465 & 0.9556 \\
\hline Calcite & 0.0598 & 0.0225 & 0.9692 \\
\hline
\end{tabular}

As seen in Table 1, all SAM values, except for the laccolite site, were small, rounding to 0.05 , the RMSE values were lower than 0.06 , and the $\mathrm{R}^{2}$ values were high $(0.90-0.98)$. This indicates high spectral similarity between DESIS and AisaFENIX and ensures good performance of the former above $450 \mathrm{~nm}$. 


\subsection{Comparison of thematic mineral mapping: DESIS vs. AisaFENIX}

This section compares iron-oxide mineral (goethite and hematite) mapping using DESIS L2A and the AisaFENIX results (resized to $30-\mathrm{m}$ spatial resolution). The matched filter method was used (Boardman, 1993). Figures 6 and 7 show the results of the DESIS thematic mapping overlaid on the MR geological map. The iron oxides are seen to be mapped in the correct geological formation of Mahmal and Inmar (blue colors in the image) (Nevo, 1963).

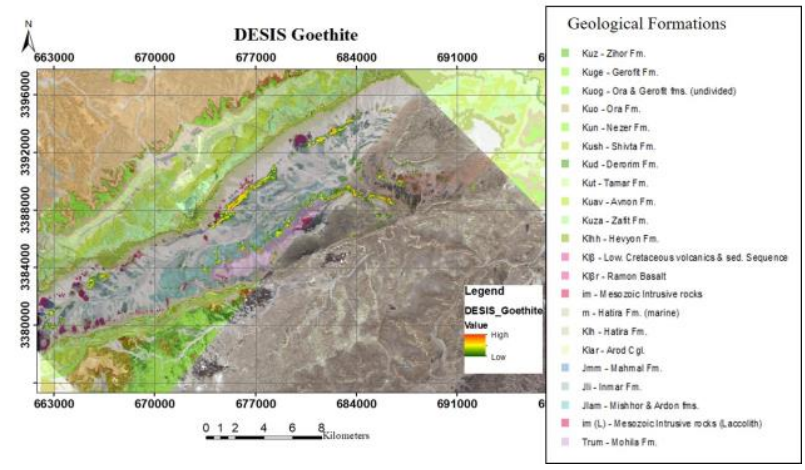

Figure 6. Goethite mapping on DESIS

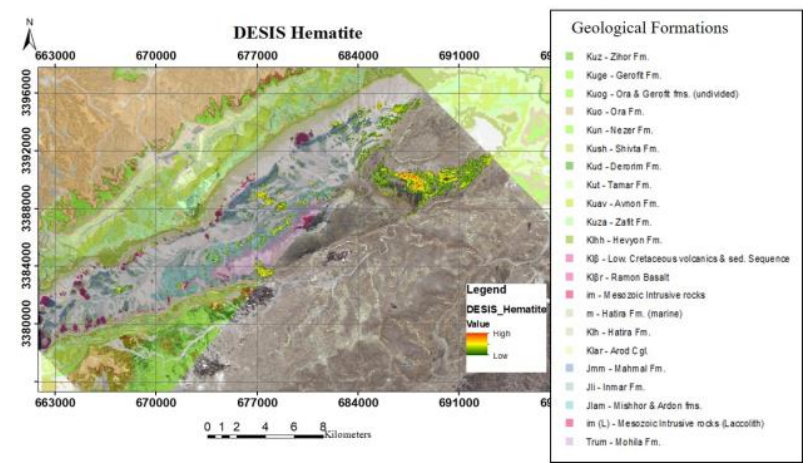

Figure 7. Hematite mapping on DESIS

Figures 8-11 show comparisons of AisaFENIX (blue) to DESIS (red) for goethite (Figures 8 and 9) and hematite (Figures 10 and 11) mapping. DESIS detected goethite and hematite in the correct geological formations, Mahmal and Inmar. Since AisaFENIX thematic maps were originally created at $1.5-\mathrm{m}$ GSD and then resized to $30 \mathrm{~m}$ for the comparison, the mapping is more accurate than the DESIS $30-\mathrm{m}$ mixed pixels, as seen in the overlay Figures 8 and 10, where some of the minerals (mostly lower concentration) were detected only by AisaFENIX (blue pixels). Nevertheless, in the areas that were zoomed-in on, there is a high similarity between the results for goethite at the brown questa (Figure 9) and hematite in Shen Ramon (Figure 11).

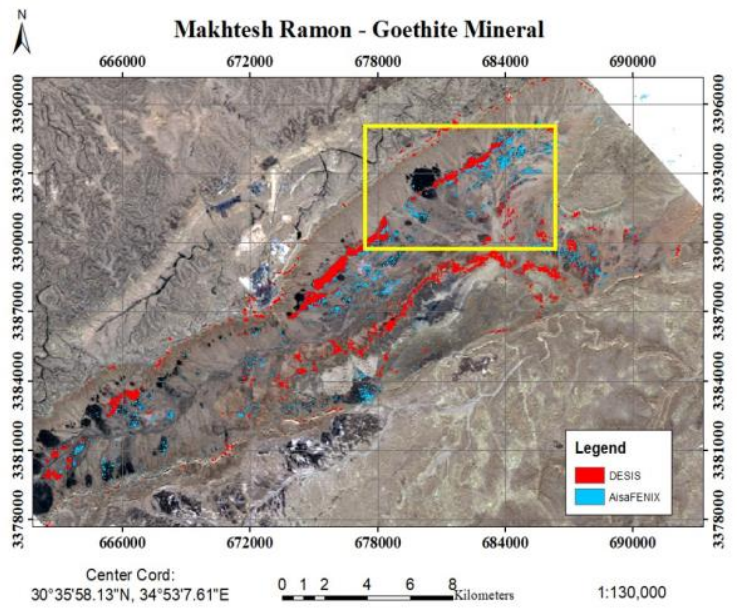

Figure 8. Goethite mapping with DESIS (red) overlaid on the AisaFENIX results (blue). The marked rectangle is the area that is zoomed-in on in Figure 9

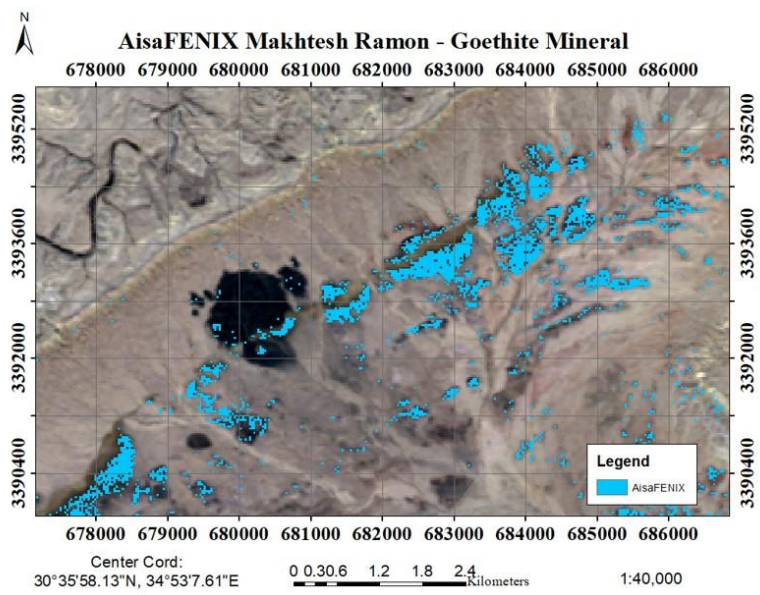

(a)

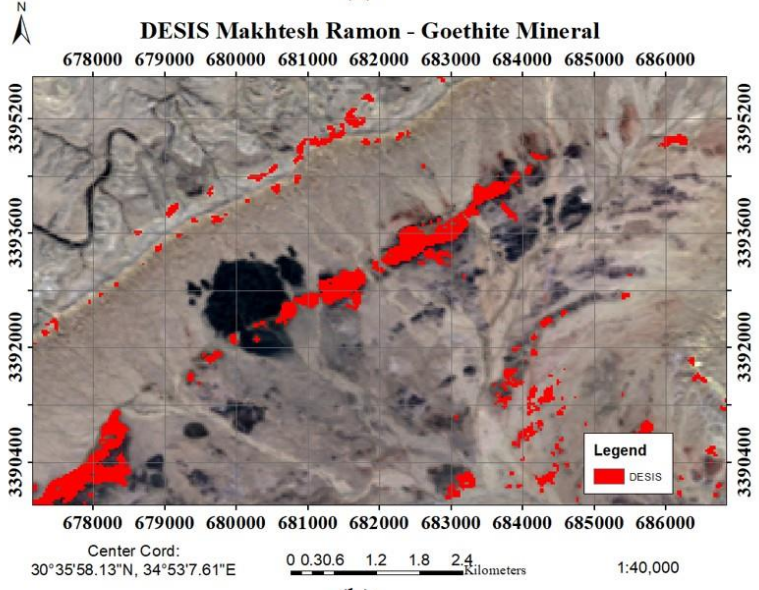

(b)

Figure 9. Goethite mapping with AisaFENIX (a) and DESIS (b); zoom-in on the area marked in Figure 8 


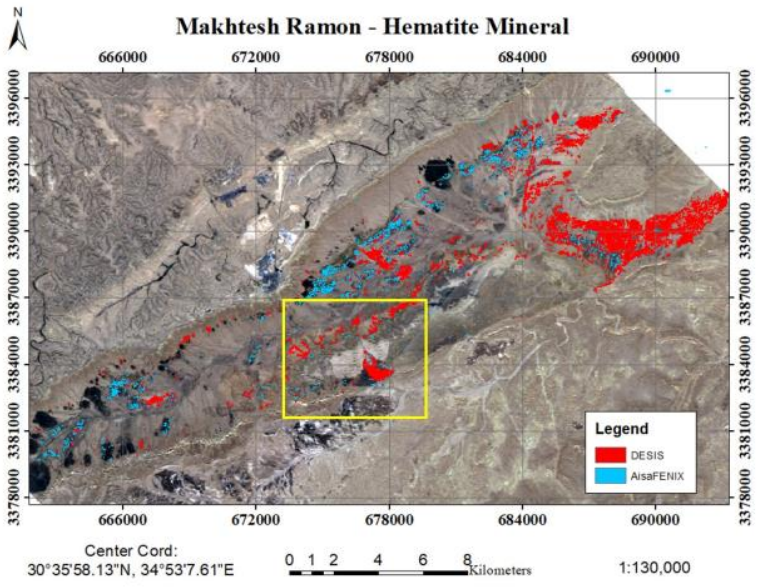

Figure 10. Hematite mapping with DESIS (red) overlaid on the AisaFENIX results (blue). The marked rectangle is the area that is zoomed-in on in Figure 11

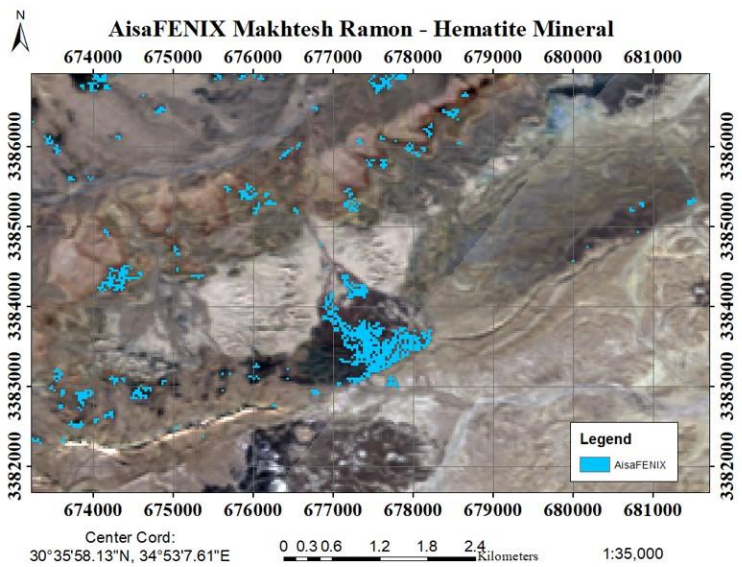

(a)

A DESIS Makhtesh Ramon - Hematite Mineral

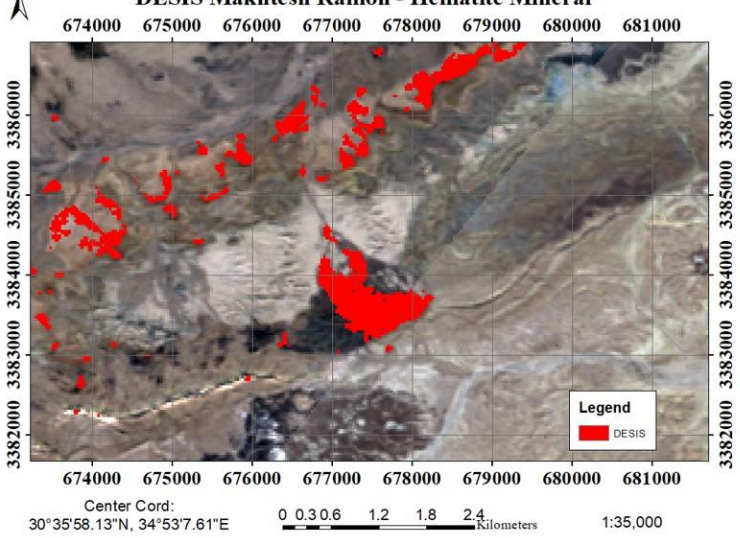

(b)

Figure 11. Hematite mapping with AisaFENIX (a) and DESIS (b); zoom-in on the area marked in Figure 10

\subsection{Comparison of thematic mineral mapping: DESIS vs. PRISMA}

This section compares the results of iron-oxide mineral mapping with DESIS L2A and another orbital sensor, the PRISMA L2D of ASI. Figures 12 and 13 compare PRISMA (green) to DESIS (red) goethite mapping and Figures 14 and 15 , their respective hematite maps.

The results for goethite and hematite are similar. It can be seen, especially in overlaid Figures 12 and 14, that the detection of pixels for each mineral is almost the same. The results for DESIS are a little more accurate for the hematite mapping. This might be due to the difference in the sensors' spectral resolution for iron-oxide mapping (400-1000) nm)- 235 DESIS bands vs. 70 PRISMA bands.

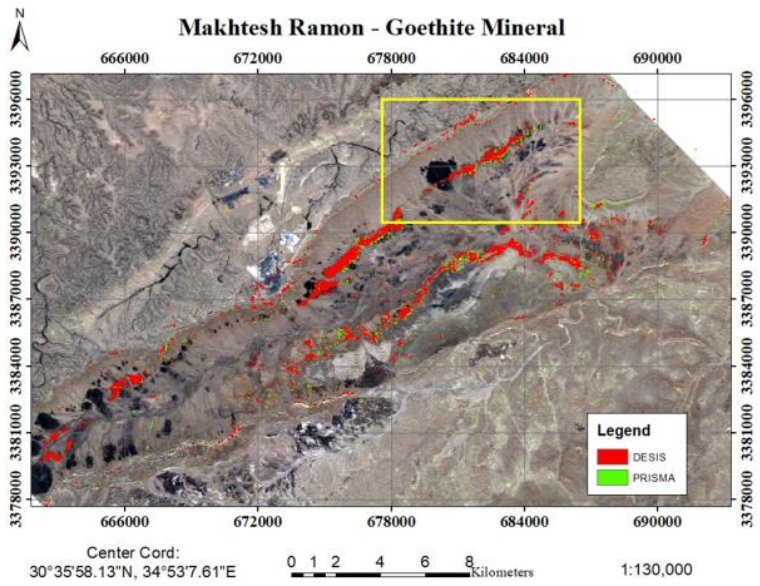

Figure 12. Goethite mapping with DESIS (red) overlaid on the PRISMA results (green). The marked rectangle is the zoomedin on the area shown in Figure13 


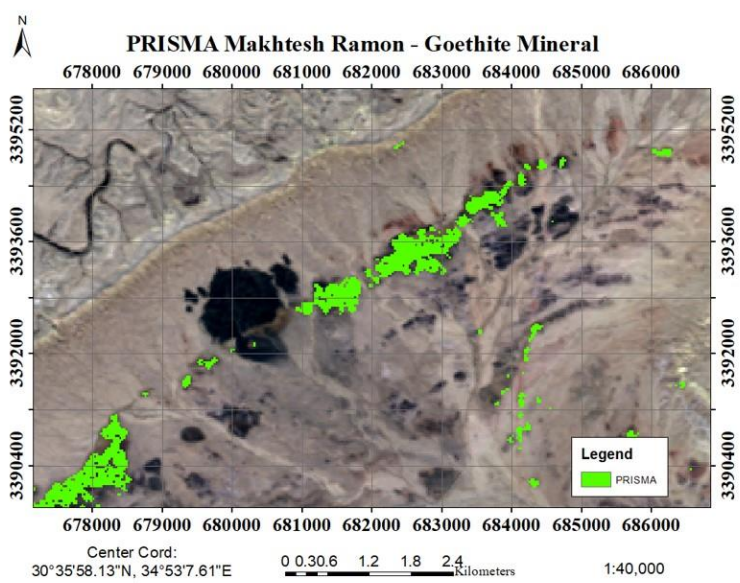

(a)

\section{$\bigwedge^{N} \quad$ DESIS Makhtesh Ramon - Goethite Mineral} 678000679000680000681000682000683000684000685000686000

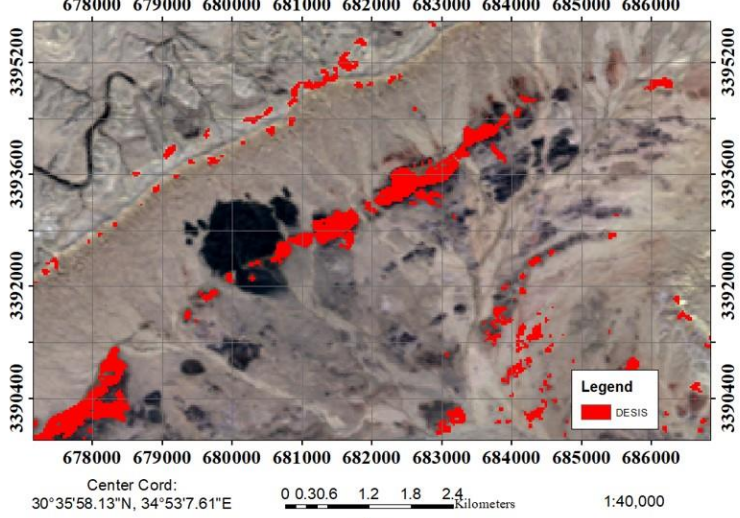

(b)

Figure 13. Goethite mapping with PRISMA (a) vs. DESIS (b). Zoom-in on the area marked in Figure 12

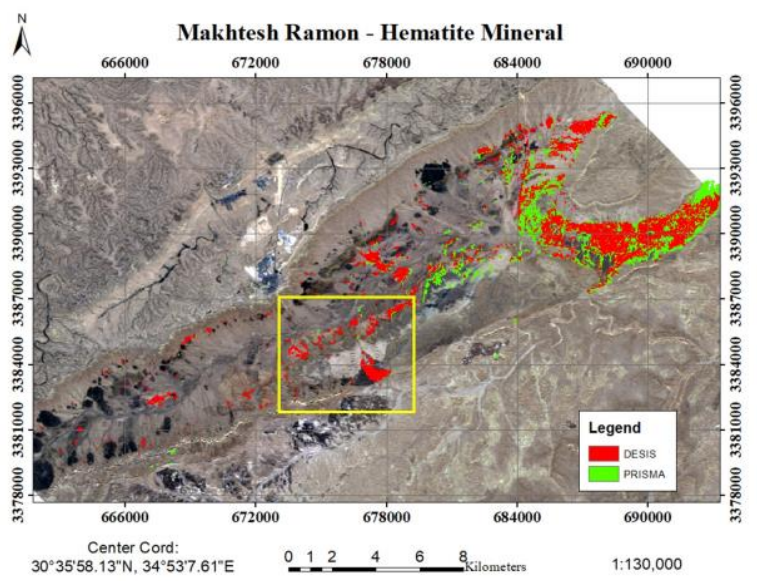

Figure 14. Hematite mapping with DESIS (red) overlaid on the PRISMA results (green). The marked rectangle is the zoomedin on area shown in Figure 15

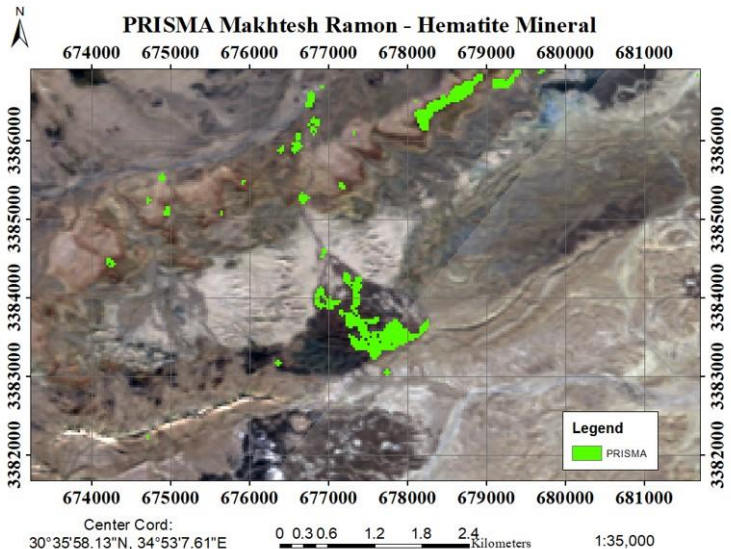

(a)

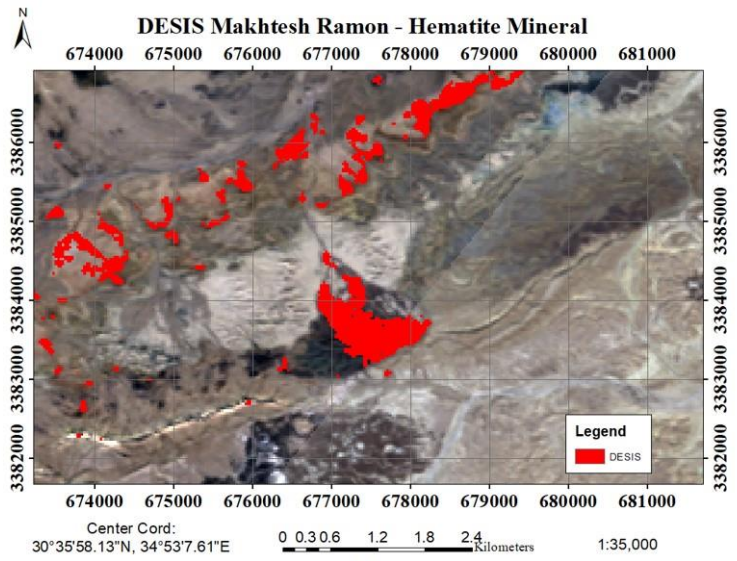

(b)

Figure 15. Hematite mapping with PRISMA (a) vs. DESIS (b). Zoom-in on the area marked in Figure 14

\section{CONCLUSIONS}

With its 235 bands and 30-m GSD, the DESIS sensor is well calibrated in both the radiometric and spectral domains. The MODTRAN simulation for AP vs. the DESIS L1C showed signal similarity regarding the spectral, radiometric shape. We demonstrated that the DESIS sensor provides a very accurate spectral-based mapping of iron-oxide minerals goethite and hematite over the MR area. We applied our VC protocol and test sites on DESIS L2A data and successfully showed the high correlation between AisaFENIX and DESIS thematic products. The spectral range below $450 \mathrm{~nm}$ of the DESIS L2A is questionable, and we suggest using only the $>450 \mathrm{~nm}$ data. In comparison with the PRISMA sensor's mapping capabilities, both sensors also behaved very similarly across the VNIR spectral region. We can conclude that the DESIS performs quite well, and further study to track the temporal stability of the sensor over MR and AP is warranted.

\section{REFERENCES}

Anker, Y., Ben Dor, E., Zelikman, E., Karnieli, A., Mazor, E., 2009. Makhtesh Ramon, a super site for calibration and validation of IS sensors. Proc. 6th EARSel Work. 3078-3084. 
Ben-Dor, E., Kruse, F.A., 1995. Surface mineral mapping of Makhtesh Ramon Negev, Israel using GER 63 channel scanner data. Int. J. Remote Sens. 18, 3529-3553.

Biggar, S.F., Slater, P.N., Gellman, D.I., 1994. Uncertainties in the in-flight calibration of sensors with reference to measured ground sites in the 0.4-1.1 $\mu \mathrm{m}$ range. Remote Sens. Environ. 48, 245-252. https://doi.org/10.1016/0034-4257(94)90145-7

Boardman, J.W., 1993. Automating spectral unmixing of AVIRIS data using convex geometry concepts. Summaries of the Fourth Annual JPL Airborne Geoscience Workshop, JPL.

Bouvet, M., Thome, K., Berthelot, B., Bialek, A., CzaplaMyers, J., Fox, N.P., Goryl, P., Henry, P., Ma, L., Marcq, S., Meygret, A., Wenny, B.N., Woolliams, E.R., 2019. RadCalNet: A radiometric calibration network for earth observing imagers operating in the visible to shortwave infrared spectral range. Remote Sens. 11. https://doi.org/10.3390/RS11202401

Cal/Val Home - CalValPortal [WWW Document], n.d. URL http://calvalportal.ceos.org/home (accessed Jul 23 2021).

Candela, L., Formaro, R., Guarini, R., Loizzo, R., Longo, F., Varacalli, G., 2016. The PRISMA mission, in: International Geoscience and Remote Sensing Symposium (IGARSS). Institute of Electrical and Electronics Engineers Inc., pp. 253256. https://doi.org/10.1109/IGARSS.2016.7729057

EOWEB GeoPortal [WWW Document], n.d. URL https://eoweb.dlr.de/egp/main\#mainWindowtabExplore?service $=$ https $\% 3 \mathrm{~A} \% 2 \mathrm{~F} \% 2 \mathrm{Feoweb} . d \mathrm{lr} . \mathrm{de} \% 2 \mathrm{Fegp} \% 2 \mathrm{Flogin} \% 2 \mathrm{Fcas} \# \mathrm{ma}$ inWindowtabExplore (accessed Jul 23 2021).

Feingersh, T., Dor, E. Ben, 2015. SHALOM - A Commercial Hyperspectral Space Mission. Opt. Payloads Sp. Mission. 247263. https://doi.org/10.1002/9781118945179.CH11

Flittner, D.E., Slater, P.N., 1991. Stability of narrow-band filter radiometers in the solar-reflective range. Photogramm. Eng. Remote Sens. 57, 165-171.

Gilead, U., Karnieli, A., 2004. Locating potential vicarious calibration sites for high-spectral resolution sensors in the Negev Desert by GIS analysis. In: Morain, A., Budge, A.M. (eds), Post-Launch Calibration of Satellite Sensors. Taylor \& Francis Group, London, pp. 181-187.

Guanter, L., Kaufmann, H., Segl, K., Foerster, S., Rogass, C., Chabrillat, S., Kuester, T., Hollstein, A., Rossner, G., Chlebek, C., Straif, C., Fischer, S., Schrader, S., Storch, T., Heiden, U., Mueller, A., Bachmann, M., Mühle, H., Müller, R., Habermeyer, M., Ohndorf, A., Hill, J., Buddenbaum, H., Hostert, P., Van Der Linden, S., Leitão, P.J., Rabe, A., Doerffer, R., Krasemann, H., Xi, H., Mauser, W., Hank, T., Locherer, M., Rast, M., Staenz, K., Sang, B., 2015. The EnMAP spaceborne imaging spectroscopy mission for earth observation. Remote Sens. 7, 8830-8857. https://doi.org/10.3390/rs70708830

Heller-Pearlshtien, D., Pignatti, S., Greisman-ran, U., Ben-Dor, E., 2021. PRISMA sensor evaluation: a case study of mineral mapping performance over Makhtesh Ramon, Israel. Int. J. Remote Sens. 42, 5882-5914.
HISUI [WWW Document], n.d. URL https://www.jspacesystems.or.jp/en/project/observation/hisui/ (accessed Jul 25 2021).

Hyperspectral Sensor AisaFENIX - Specim, Spectral Imaging Ltd. [WWW Document], n.d. URL https://www.specim.fi/products/aisafenix-hyperspectral-sensor/ (accessed Jul 25 2021).

HySIS [WWW Document], n.d. URL https://directory.eoportal.org/web/eoportal/satellitemissions/content/-/article/hysis (accessed Jul 25 2021).

Kabir, S., Leigh, L., Helder, D., 2020. Vicarious methodologies to assess and improve the quality of the optical remote sensing images: A critical review. Remote Sens. 12, 1-40. https://doi.org/10.3390/rs12244029

Kamei, A., Nakamura, K., Tachikawa, T., Yamamoto, H., Nakamura, R., Tsuchida, S., 2011. The long-term vicarious and cross calibration plan for Hyper-spectral Imager Suite (HISUI). Int. Geosci. Remote Sens. Symp. 1013-1016. https://doi.org/10.1109/IGARSS.2011.6049305

Kaufman, H., 1991. Mineral Identification Using GER-II data acquired from Makhtesh Ramon/Negev, Israel. EARSeL Adv. Remote Sens. 1, 82-92.

Kruse, F.A., Lefkoff, A.B., Boardman, J.W., Heidebrecht, K.B., Shapiro, A.T., Barloon, P.J., Goetz, A.F.H., 1993. The spectral image processing system (SIPS) - interactive visualization and analysis of imaging spectrometer data. Remote Sens. Environ. 44, 145-163. https://doi.org/10.1016/0034-4257(93)90013-N

Krutz, D., Müller, R., Knodt, U., Günther, B., Walter, I., Sebastian, I., Säuberlich, T., Reulke, R., Carmona, E., Eckardt, A., Venus, H., Fischer, C., Zender, B., Arloth, S., Lieder, M., Neidhardt, M., Grote, U., Schrandt, F., Gelmi, S., Wojtkowiak, A., 2019. The instrument design of the DLR earth sensing imaging spectrometer (DESIS). Sensors (Switzerland) 19. https://doi.org/10.3390/s19071622

Liu, Y.N., Sun, D.X., Hu, X.N., Liu, S.F., Cao, K.Q., 2020. AHSI: the hyperspectral imager on China's GaoFen-5 Satellite, in: IOP Conference Series: Earth and Environmental Science. Institute of Physics Publishing, p. 012033. https://doi.org/10.1088/1755-1315/509/1/012033

Makhtesh Ramon Cal/Val Site [WWW Document], n.d. URL https://storymaps.arcgis.com/stories/bb5bf09ec7414454a012bfe 9bf4b8545 (accessed Jul 232021

MODTRAN ${ }^{\circledR} \quad$ [WWW Document], n.d. URL http://modtran.spectral.com/modtran_index (accessed 9.23.21).

Müller, R., 2014. Calibration and verification of remote sensing instruments and observations. Remote Sens. 6, 5692-5695. https://doi.org/10.3390/rs6065692

Nevo, E., 1963. The Jurassic strata of Makhtesh Ramon. Proceedings in Ramon Symposium. The Israel Geological Society (GSI), Jerusalem, Israel.

Notesco, G., Ogen, Y., Ben-Dor, E., 2015. Mineral classification of Makhtesh Ramon in Israel using hyperspectral 
longwave infrared (LWIR) remote-sensing data. Remote Sens. 7, 12282-12296. https://doi.org/10.3390/rs70912282

Notesco, G., Ogen, Y., Ben-Dor, E., 2016. Integration of hyperspectral shortwave and longwave infrared remote-sensing data for mineral mapping of Makhtesh Ramon in Israel. Remote Sens. 8. https://doi.org/10.3390/rs8040318

Obata, K., Tsuchida, S., Yamamoto, H., Thome, K., 2017.

Cross-calibration between ASTER and MODIS visible to nearinfrared bands for improvement of aster radiometric calibration. Sensors (Switzerland) 17. https://doi.org/10.3390/s17081793

PRISMA [WWW Document], n.d. URL http://prismai.it/index.php/en/ (accessed Mar 22 2021).

Robert, S.F., Kaufman, Y.J., 1986. Calibration of satellite sensors after launch. Appl. Opt. 25, 1177-1185.

Schmidt, H., Karnieli, A., 2001. Sensitivity of vegetation indices to substrate brightness in hyper-arid environment: the Makhtesh Ramon Crater (Israel) case study. Int. J. Remote Sens. 22, 3503-3520. https://doi.org/10.1080/01431160110063779

Scott, K.P., Thome, K.J., Brownlee, M.R., 1996. Evaluation of Railroad Valley playa for use in vicarious calibration. In Huberty, B., Lurie, J.B., Caylor, J.A., Coppin, P., Robert, P.C. (eds.), Multispectral Imaging for Terrestrial Applications. SPIE's 1996 International Symposium on Optical Science, Engineering, and Instrumentation, Denver, CO, p. 158. https://doi.org/10.1117/12.256090

Secker, J., Staenz, K., Gauthier, R.P., Budkewitsch, P., 2001. Vicarious calibration of airborne hyperspectral sensors in operational environments. Remote Sens. Environ. 76, 81-92. https://doi.org/10.1016/S0034-4257(00)00194-2

Suits, G., Malila, W., Weller, T., 1988. The prospects for detecting spectral shifts due to satellite sensor aging. Remote Sens. Environ. 26, 17-29. https://doi.org/10.1016/00344257(88)90117-4

Teillet, P.M., 2015. Overview of satellite image radiometry in the solar-reflective optical domain. In Thenkabail, P.S. (ed.), Remotely Sensed Data Characterization, Classification, and Accuracies. CRC Press, Boca Raton. https://doi.org/10.1201/b19294

Teillet, P.M., Slater, P.N., Ding, Y., Santer, R.P., Jackson, R.D., Moran, M.S., 1990. Three methods for the absolute calibration of the NOAA AVHRR sensors in-flight. Remote Sens. Environ. 31, 105-120. https://doi.org/10.1016/00344257(90)90060-Y

Thenkabail, P., 2016. Remote Sensing Handbook Volume 1: Remotely Sensed Data Characterized, Classification and Accuracies. Taylor \& Francis Group, London.

Yoshida, M., Murakami, H., Mitomi, Y., Hori, M., Thome, K.J., Clark, D.K., Fukushima, H., 2005. Vicarious calibration of GLI by ground observation data. IEEE Trans. Geosci. Remote Sens. 43, 2167-2175. https://doi.org/10.1109/TGRS.2005.856113 